\title{
Surface Tension Calculation of Mixed Solvents with Respect to Solvent Composition and Temperature by Using Jouyban-Acree Model
}

\author{
Abolghasem Jouyban, ${ }^{* a}{ }^{a}$ Anahita Fathi-Azarbayjani, ${ }^{b}$ and William Eugene Acree, $\mathrm{Jr}^{c}$ \\ ${ }^{a}$ School of Pharmacy and Drug Applied Research Center, Tabriz University of Medical Sciences; Tabriz 51664, Iran: \\ ${ }^{b}$ Kimia Research Institute; Tabriz, P.O. Box 51665-171, Iran: and ${ }^{c}$ Department of Chemistry, University of North Texas; \\ Denton, TX 76203-5070, U.S.A. Received June 23, 2004; accepted July 15, 2004
}

\begin{abstract}
Applicability of a solution model, i.e. Jouyban-Acree model (JAM), for calculating surface tension of binary and ternary solvents at various temperatures has been shown employing experimental surface tension data collected from the literature. The accuracy of the model was evaluated by calculating average percentage deviation (APD) between calculated and observed values. The obtained overall APD ( \pm S.D.) for JAM using binary solvent data were $4.06( \pm 4.27)$ and $8.07( \pm 9.78) \%$, respectively for correlative and predictive analyses. The corresponding values for the best similar model from the literature were $8.86( \pm 6.40)$ and $37.10( \pm 27.65) \%$ and the mean APD differences between JAM and previously published model were significant $(p<0.003)$. The capability of JAM for correlating surface tension of ternary solvents at various temperatures was also shown and the overall APD was $1.39( \pm 0.37) \%$.
\end{abstract}

Key words surface tension; mixed solvent; mathematical model; prediction

Surface and/or interfacial tension is a measurable quantity, which represents the surface and/or interfacial activity and differs from the assumed ideal value when the system becomes non-ideal. Surface tension plays an important role in the functioning of respiratory system. Surface tension of the fluid lining the alveoli is decreased by a mixture of surface active agents mainly, dipalmitoyl lecithin. Respiratory distress syndrome and atelectasis are two major clinical syndromes of increasing the surface tension of the fluid lining the alveoli. ${ }^{1)}$ Prokop et $a .^{2)}$ reported the relationships between recoil pressure and surface area of the lung and the surface tension of the lung fluid. Several important phenomena in the pharmaceutical and analytical areas are related to the surface activity. This includes suspension and emulsion formulations, solubilization techniques and explaining the retention mechanisms in HPLC. The primary droplet size of the nebulized solutions was correlated to the surface tension of the solution. ${ }^{3)}$ Binary and ternary solvents have been used in many pharmaceutical formulations and also validated analytical methods at ambient and higher and/or lower temperatures. The surface tension data of mixed solvents are required in practice and the aim of this work is to present a simple mathematical model for calculating such data.

\section{Computational Methods}

The basic solution model, i.e. the combined nearly ideal binary solvent/Redlich-Kister equation (CNIBS/R-K), presented by Acree ${ }^{4)}$ was used to correlate different physico-chemical properties in mixed solvent systems; including the solubility of drugs in water-cosolvent mixtures, ${ }^{5}{ }^{5}$ electrophoretic mobility of analytes in mixed solvent electrolyte systems, ${ }^{6-8)}$ the instability rate constants in binary solvent systems, ${ }^{9}{ }^{9}$ the acid dissociation constants in water-organic solvent mixtures ${ }^{10)}$ the dielectric constant ${ }^{11)}$ and surface tension ${ }^{12)}$ of solvent mixtures. Theoretical basis of the CNIBS/R-K equation for describing the chemical potential of solutes dissolved in mixed solvents ${ }^{4)}$ and acid dissociation constants in aqueous-organic mixtures ${ }^{10)}$ have been provided in earlier papers.

The Jouyban-Acree model (JAM) representing the surface tension of binary solvent mixtures at a constant temperature has been proposed in our earlier work $^{12)}$ as:

$$
\ln \gamma_{\mathrm{m}}=\phi_{1} \ln \gamma_{1}+\phi_{2} \ln \gamma_{2}+\phi_{1} \phi_{2} \sum_{i=0}^{2} K_{i}\left(\phi_{1}-\phi_{2}\right)^{i}
$$

Where $\gamma_{\mathrm{m}}, \gamma_{1}$ and $\gamma_{2}$ are the surface tensions of the mixture and solvents 1 and 2, respectively, $\phi_{1}$ and $\phi_{2}$ are the volume (weight or mole) fractions of solvents 1 and 2 in the mixture and $K_{\mathrm{i}}$ represent the model constants calculated using a least square method. ${ }^{13)}$ The model could be extended for calculating the surface tension of ternary solvents as:

$$
\begin{aligned}
\ln \gamma_{\mathrm{m}}= & \phi_{1} \ln \gamma_{1}+\phi_{2} \ln \gamma_{2}+\phi_{3} \ln \gamma_{3}+\phi_{1} \phi_{2} \sum_{i=0}^{2} K_{i}\left(\phi_{1}-\phi_{2}\right)^{i} \\
& +\phi_{1} \phi_{3} \sum_{i=0}^{2} K_{i}^{\prime}\left(\phi_{1}-\phi_{3}\right)^{i}+\phi_{2} \phi_{3} \sum_{i=0}^{2} K_{i}^{\prime \prime}\left(\phi_{2}-\phi_{3}\right)^{i} \\
& +\phi_{1} \phi_{2} \phi_{3} \sum_{i=0}^{2} K_{i}^{\prime \prime \prime}\left(\phi_{1}-\phi_{2}-\phi_{3}\right)^{i}
\end{aligned}
$$

Where $K_{i}, K_{i}^{\prime}, K_{i}^{\prime \prime}$ and $K_{i}^{\prime \prime \prime}$ are the model constants. ${ }^{12)}$

To correlate the surface tensions of binary solvents at various temperatures $(T)$, an extended form of JAM could be used for calculating the surface tension with respect to solvent composition and temperature, and is proposed in this work as:

$$
\ln \gamma_{\mathrm{m}, T}=\phi_{1} \ln \gamma_{1, T}+\phi_{2} \ln \gamma_{2, T}+\phi_{1} \phi_{2} \sum_{j=0}^{2}\left[\frac{A_{j}\left(\phi_{1}-\phi_{2}\right)^{j}}{T}\right]
$$

Where $\gamma_{\mathrm{m}, T}, \gamma_{1, T}$ and $\gamma_{2, T}$ are the surface tensions of the mixture and solvents 1 and 2 at temperature $T$, respectively, and $A_{j}$ represent the model constants. The proposed model could also be extended to ternary solvents at different temperatures as:

$$
\begin{aligned}
\ln \gamma_{\mathrm{m}, T}= & \phi_{1} \ln \gamma_{1, T}+\phi_{2} \ln \gamma_{2, T}+\phi_{3} \ln \gamma_{3, T}+\phi_{1} \phi_{2} \sum_{j=0}^{2}\left[\frac{A_{j}\left(\phi_{1}-\phi_{2}\right)^{j}}{T}\right] \\
& +\phi_{1} \phi_{3} \sum_{j=0}^{2}\left[\frac{B_{j}\left(\phi_{1}-\phi_{3}\right)^{j}}{T}\right]+\phi_{2} \phi_{3} \sum_{j=0}^{2}\left[\frac{C_{j}\left(\phi_{2}-\phi_{3}\right)^{j}}{T}\right] \\
& +\phi_{1} \phi_{2} \phi_{3} \sum_{j=0}^{2}\left[\frac{D_{j}\left(\phi_{1}-\phi_{2}-\phi_{3}\right)^{j}}{T}\right]
\end{aligned}
$$

Where $\gamma_{3, T}$ is the surface tension of the solvent 3 at temperature $T, \phi_{3}$ denotes the volume (weight or mole) fraction of the third solvent and $A_{j}$, $B_{j}, C_{j}$ and $D_{j}$ are the model constants. These model constants are computed by regressing $\left(\ln \gamma_{\mathrm{m}, T}-\phi_{1} \ln \gamma_{1, T}-\phi_{2} \ln \gamma_{2, T}-\phi_{3} \ln \gamma_{3, T}\right) \quad$ against $\phi_{1} \phi_{2} / T$, $\phi_{1} \phi_{2}\left(\phi_{1}-\phi_{2}\right) / T, \phi_{1} \phi_{2}\left(\phi_{1}-\phi_{2}\right)^{2} / T, \phi_{1} \phi_{3} / T, \phi_{1} \phi_{3}\left(\phi_{1}-\phi_{3}\right) / T, \phi_{1} \phi_{3}\left(\phi_{1}-\phi_{3}\right)^{2} / T$, $\phi_{2} \phi_{3} / T, \phi_{2} \phi_{3}\left(\phi_{2}-\phi_{3}\right) / T, \phi_{2} \phi_{3}\left(\phi_{2}-\phi_{3}\right)^{2} / T, \phi_{1} \phi_{2} \phi_{3} / T, \phi_{1} \phi_{2} \phi_{3}\left(\phi_{1}-\phi_{2}-\phi_{3}\right) / T$ and $\phi_{1} \phi_{2} \phi_{3}\left(\phi_{1}-\phi_{2}-\phi_{3}\right)^{2} / T$ using a no intercept least square analysis.

Connors and Wright ${ }^{14)}$ have proposed Eq. 5 for calculating surface tension 
of mixed solvents at a constant temperature. Eq. 5 is:

$$
\gamma_{\mathrm{m}}=\gamma_{1}-\left[1+\frac{b \phi_{1}}{1-a \phi_{1}}\right] \phi_{2}\left(\gamma_{1}-\gamma_{2}\right)
$$

where $a$ and $b$ are the model constants. Hoke and Patton ${ }^{15)}$ have also correlated Connors model constants with temperature using:

$$
a=M_{0}+M_{1} T+M_{2} T^{2}
$$

and

$$
b=P_{0}+P_{1}+P_{2} T^{2}
$$

By combining Connors model with Hoke and Patton's equation, the following equation could be obtained:

$$
\gamma_{\mathrm{m}}=\gamma_{1}-\left[1+\frac{H_{1} \phi_{1}+H_{2} \phi_{1} T+H_{3} \phi_{1} T^{2}}{1-\left(H_{4} \phi_{1}+H_{5} \phi_{1} T+H_{6} \phi_{1} T^{2}\right)}\right] \phi_{2}\left(\gamma_{1}-\gamma_{2}\right)
$$

in which $H_{1}-H_{6}$ are the model constants. This combination enables Connors' model to calculate surface tension at different temperatures and solvent compositions.

Lee et al. ${ }^{16)}$ have used the following equation for calculating surface tension of solvent mixtures based on solvent composition and temperature:

$$
\gamma_{\mathrm{m}, T}=\sum_{i=0}^{2}\left[L_{i} \phi_{1}^{i}+L_{i}^{\prime}\left(\phi_{1}^{i} \cdot T\right)+L_{i}^{\prime \prime}\left(\phi_{1}^{i} \cdot T^{2}\right)\right]
$$

where $L_{i}, L_{i}{ }^{\prime}$ and $L_{i}^{\prime \prime}$ are the model constants calculated using a least square analysis. ${ }^{16)}$ Lee's model is based on mixture response method and could be rewritten as:

$$
\begin{aligned}
\gamma_{\mathrm{m}, T}= & M_{0}+M_{1} \phi_{1}+M_{2} \phi_{1}^{2}+M_{3} T+M_{4} \phi_{1} T+M_{5} \phi_{1}^{2} T+M_{6} T^{2}+M_{7} \phi_{1} T^{2}+ \\
& M_{8} \phi_{1}^{2} T^{2}
\end{aligned}
$$

where $M_{0}-M_{8}$ are the model constants.

The calculated surface tensions were compared with experimental (observed) values and the absolute percentage deviation (APD) was used as an accuracy criterion. The APD was calculated using:

$$
\mathrm{APD}=\frac{100}{N} \sum \frac{\mid \text { calculated }- \text { observed } \mid}{\text { observed }}
$$

where $N$ is the number of data points in each set. The overall APD (OAPD) was defined as:

$$
\text { OAPD }=\frac{\sum \text { APD }}{\text { number of data sets studied }}
$$

and was used as overall accuracy criterion for comparing different models.
Individual percentage deviation (IPD) is another criterion to check the suitability of a model in practice and calculated as:

$$
\operatorname{IPD}=100\left(\frac{\mid \text { calculated }- \text { observed } \mid}{\text { observed }}\right)
$$

\section{Results and Discussion}

The available experimental surface tension of aqueous binary solvents at various temperatures were fitted to JAM and Eqs. 8 and 10 and the APD values were computed using back-calculated surface tensions. The details of the data, the number of data points, temperature ranges, surface tension ranges in water and cosolvent and the APD values were listed in Table 1. The JAM produced the minimum and maximum APD values respectively for water-1,3-propandiol (0.34) and water-1-propanol (16.00). Equation 8 showed the same data sets as minimum and maximum values with APD values of 1.17 and $82.07 \%$, respectively. On the other hand, Eq. 10 correlated the surface tension data with minimum and maximum APDs for water-1,3-propandiol (0.77) and water2-propanol (22.00), respectively. The obtained OAPD and the standard deviations for JAM and Eqs. 8 and 10 are $4.06 \pm 4.27,26.88 \pm 23.85$ and $8.86 \pm 6.40$, respectively. The OAPD differences between JAM and Eqs. 8 and 10 were statistically tested using paired $t$-test and the results revealed that JAM is able to correlate surface tension of binary solvents at various temperatures more accurate than Eqs. 8 and 10. It is possible to use more curve-fitting parameters with JAM for increasing its accuracy. As a general rule for mathematical models containing constant terms, the more the number of constant terms the more accurate the results and this has been observed in this study with JAM. The OAPD and standard deviations for different numbers of curve-fitting parameters were shown in Fig. 1. The improvement in accu-

\begin{tabular}{|c|c|c|c|c|c|c|c|c|c|}
\hline No. & Cosolvent (Solvent 2) & Ref. & $N$ & $T$ range & $\gamma_{1}$ range & $\gamma_{2}$ range & JAM & Eq. 8 & Eq. 10 \\
\hline 1 & Acetone & 17 & 59 & $293-323$ & $68.0-72.7$ & $19.5-23.2$ & 6.79 & 42.31 & 15.14 \\
\hline 2 & 2-Amino-2-methyl-1-propanol & 18 & 84 & $298-323$ & $67.9-72.0$ & $28.4-31.3$ & 6.57 & 32.15 & 10.33 \\
\hline 3 & 3-Amino-1-propanol & 19 & 72 & $298-323$ & $67.9-72.0$ & $38.1-43.9$ & 1.18 & 8.83 & 3.84 \\
\hline 4 & 1-Amino-2-propanol & 19 & 72 & $298-323$ & $67.9-72.0$ & $31.2-37.3$ & 3.19 & 16.81 & 6.74 \\
\hline 5 & $\gamma$-Butyrolactone & 20 & 33 & $303-343$ & $64.3-71.2$ & $37.9-42.7$ & 1.09 & 10.88 & 5.60 \\
\hline 6 & Diethanolamine & 21 & 66 & $298-323$ & $67.9-72.0$ & $43.1-47.2$ & 1.51 & 8.85 & 3.66 \\
\hline 7 & Dioxane & 22 & 85 & $293-353$ & $62.6-72.7$ & $24.7-33.3$ & 2.65 & 25.61 & 9.52 \\
\hline 8 & Ethanol & 23 & 98 & $293-323$ & $67.9-72.7$ & $19.8-22.3$ & 4.88 & 47.21 & 16.32 \\
\hline 9 & Methanol & 24 & 40 & $293-323$ & $67.9-72.7$ & $19.5-22.6$ & 0.80 & 13.68 & 4.36 \\
\hline 10 & Methanol & 23 & 98 & $293-323$ & $67.9-72.7$ & $20.2-22.9$ & 1.36 & 26.44 & 8.13 \\
\hline 11 & $N$-Methyldiethanolamine & 25 & 84 & $298-323$ & $67.9-72.0$ & $36.8-38.9$ & 4.10 & 17.44 & 5.89 \\
\hline 12 & Monoethanolamine & 18 & 78 & $298-323$ & $67.9-72.0$ & $44.8-48.9$ & 1.59 & 7.80 & 3.04 \\
\hline 13 & 1,3-Propandiol & 16 & 42 & $298-323$ & $67.9-71.8$ & $51.1-53.1$ & 0.34 & 1.17 & 0.77 \\
\hline 14 & 1-Propanol & 23 & 98 & $293-323$ & $67.9-72.7$ & $21.3-23.7$ & 16.00 & 82.07 & 20.60 \\
\hline 15 & 2-Propanol & 23 & 98 & $293-323$ & $67.9-72.7$ & $18.7-21.7$ & 10.95 & 75.21 & 22.00 \\
\hline 16 & Triethanolamine & 23 & 66 & $298-323$ & $67.9-72.0$ & $41.8-45.9$ & 1.99 & 13.65 & 5.85 \\
\hline
\end{tabular}
racy with increase in the number of constant terms reaches a limiting value after the inclusion of about 4 or 5 curve-fitting parameters. Since one of the main purposes of modeling is to predict unmeasured quantities using minimum experimentally derived information, it is suggested to use JAM for calculating surface tension of mixed solvents with $j=2$ which needs a minimum number of five data points. The accuracy

Table 1. Details of Surface Tension of Aqueous Binary Solvent Mixtures, the References, Number of Data Points $(N)$, Temperature $(T)$ and Surface Tension Ranges for Solvents 1 and 2, and the Average Percentage Deviations (APDs) for JAM and Eqs. 8 and 10 Using Correlative Analysis 


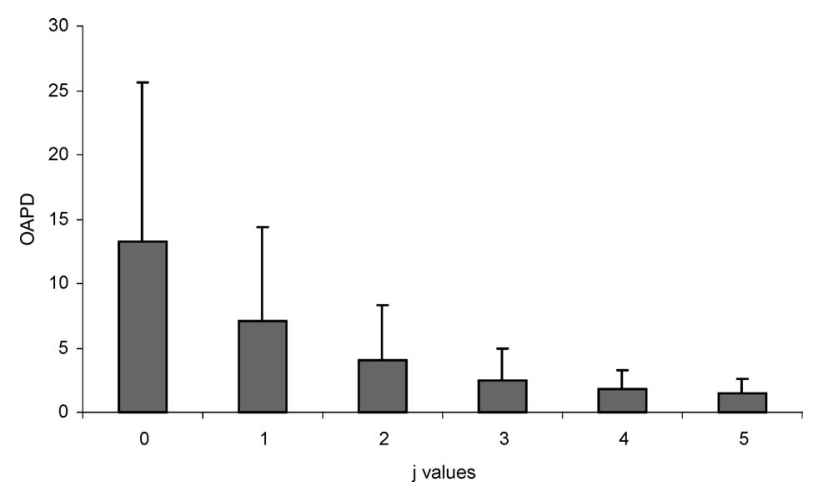

Fig. 1. OAPD $( \pm$ S.D. $)$ of JAM with Different $j$ Values for Correlating Surface Tension of Binary Solvents at Various Temperatures

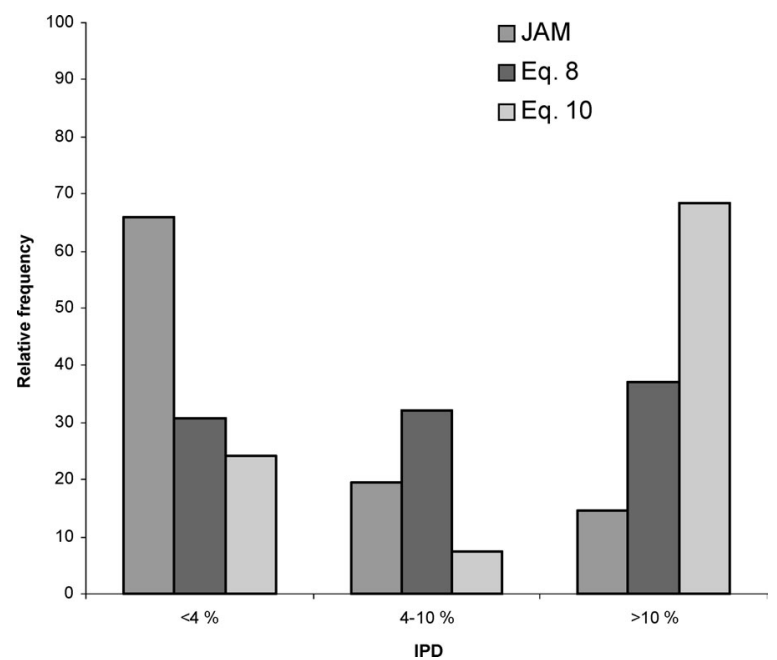

Fig. 2. IPD Distribution for Correlative Analysis of Surface Tension Data of Binary Solvents at Various Temperatures Using Three Studied Equations

of the models for calculating surface tension was also evaluated by studying the distribution of IPD for correlative studies. The IPD values sorted in three error ranges, i.e. $\leq 4,4$ 10 and $>10 \%$ and the findings were shown in Fig. 2. For JAM, more than $85 \%$ of IPD values are $\leq 10$ and only $c a$. $15 \%$ of data points produced IPD more than 10 whereas the corresponding frequencies for Eq. 10 were 32 and $68 \%$. Considering these findings, it is observed that JAM is able to provide more accurate correlation in comparison with previously published similar models.

As a general rule, a model with good correlation abilities, shows better prediction capability than that of poor correlative models. To test the prediction capability of the models and test the rule on real experimental data, a minimum number of experimental surface tensions were employed to train the models. The training points involve the surface tension of pure water and cosolvent at all temperatures under investigation and three surface tensions from binary mixtures with nearly constant volume/mole fraction intervals, i.e. $\phi_{1}=0.3$, 0.5 and 0.7 at the highest and lowest temperatures of interest. After training the models, the surface tensions at other solvent compositions and temperatures were predicted using interpolation technique. The obtained APD values were listed in Table 2. Figure 3 shows IPD distribution of the equations under investigation in three error subgroups for predictive
Table 2. Prediction Capability of Equations Studied after Training with a Minimum Number of Experimental Data Points

\begin{tabular}{ccccc}
\hline \hline No. $^{a)}$ & $N$ & JAM & Eq. 8 & Eq. 10 \\
\hline 1 & 45 & 9.15 & 94.00 & 57.36 \\
2 & 66 & 14.80 & 139.86 & 42.09 \\
3 & 54 & 1.99 & 131.86 & 11.09 \\
4 & 54 & 3.18 & 96.59 & 20.25 \\
5 & 17 & 2.35 & 125.57 & 39.24 \\
6 & 48 & 3.29 & 923.16 & 12.29 \\
7 & 63 & 4.39 & 162.52 & 33.09 \\
8 & 78 & 10.22 & 87.33 & 53.22 \\
9 & 26 & 1.33 & 116.56 & 36.48 \\
10 & 78 & 2.19 & 110.25 & 40.63 \\
11 & 66 & 7.42 & 282.09 & 23.16 \\
12 & 60 & 2.47 & 300.18 & 9.50 \\
13 & 24 & 0.48 & 125.59 & 3.91 \\
14 & 78 & 35.35 & 79.62 & 103.15 \\
15 & 78 & 26.13 & 155.31 & 86.94 \\
16 & 48 & 4.37 & 115.49 & 21.12 \\
& & $8.07 \pm 9.78^{* * * *}$ & $190.37 \pm 205.31^{*}$ & $37.10 \pm 27.65^{* *}$ \\
\end{tabular}

a) The key for the numbers is the same as in Table $1 . *, * *$ The mean differences between JAM and the previous models are statistically significant (paired $t$-test, $p<0.003)$.

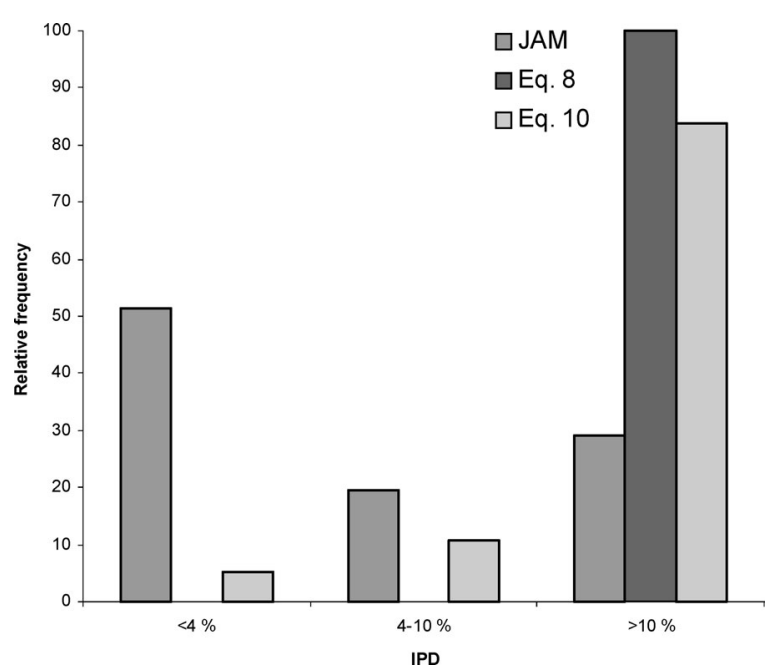

Fig. 3. IPD Distribution for Predictive Analysis of Surface Tension Data of Binary Solvents at Various Temperatures Using Three Studied Equations

analysis. The relative frequency of IPDs $<10 \%$ for JAM and Eqs. 8 and 10 are 71,0 and $16 \%$, respectively. This means that using JAM and minimum number of data points, it is possible to predict unmeasured surface tensions and the probability of prediction error less than $10 \%$ is 0.70 whereas for Eq. 8 all predicted points produced prediction errors more than 10 and the corresponding probability for Eq. 10 is 0.16 .

Applicability of JAM for correlating the surface tension of ternary solvent mixtures at various temperatures has been evaluated using 10 experimental data sets. The details of data sets and APD values were listed in Table 3. As seen from low OAPD value, JAM is capable of providing accurate correlation for surface tension of ternary solvents at various temperatures and it is expected to be an accurate model for predicting surface tension of ternary solvents using a minimum number of experimental data. To the best of our knowledge, there is no such a similar model in the literature to compare 
Table 3. Details of Surface Tension of Aqueous Ternary Solvent Mixtures, the References, Number of Data Points $(N)$, Temperature $(T)$ Ranges and Average Percentage Deviation (APD), Overall APD (OAPD) and Its Standard Deviation Values for JAM

\begin{tabular}{|c|c|c|c|c|c|}
\hline No. & Solvent (2) + Solvent (3) & Ref. & $N$ & $T$ range & APD \\
\hline 1 & 3-Amino-1-propanol+2-amino-2-methyl-1-propanol & 19 & 36 & $298-323$ & 1.38 \\
\hline 2 & 1-Amino-2-propanol+2-amino-2-methyl-1-propanol & 19 & 36 & $298-323$ & 1.96 \\
\hline 3 & 3-Amino-1-propanol+diethanolamine & 19 & 36 & $298-323$ & 0.99 \\
\hline 4 & 1-Amino-2-propanol+diethanoleamine & 19 & 36 & $298-323$ & 1.59 \\
\hline 5 & 3-Amino-1-propanol+triethanolamine & 19 & 36 & $298-323$ & 1.28 \\
\hline 6 & 1-Amino-2-propanol+triethanolamine & 19 & 36 & $298-323$ & 1.85 \\
\hline 7 & $N$-Methyldiethanolamine +2 -amino-2-methyl-1-propanol & 25 & 36 & $298-323$ & 1.27 \\
\hline 8 & $N$-Methyldiethanolamine + diethanoleamine & 25 & 36 & $298-323$ & 1.27 \\
\hline 9 & $N$-Methyldiethanolamine + monoethanolamine & 25 & 36 & $298-323$ & 0.73 \\
\hline \multirow[t]{2}{*}{10} & Monoethanolamine +2 -amino-2-methyl-1-propanol & 18 & 36 & $298-323$ & 1.54 \\
\hline & & & & OAPD \pm S.D. & $1.39 \pm 0.37$ \\
\hline
\end{tabular}

its accuracy with JAM.

\section{Conclusion}

JAM produced accurate calculations for surface tension data of mixed solvent systems at various temperatures. Its accuracy was better than those of similar mathematical models from the literature for calculating surface tension of binary solvents. The binary and ternary systems considered in the present communication were limited to those mixtures containing water as one of the solvent components as we wanted to test the descriptive/predictive ability of JAM on systems covering as wide of a range of surface tensions as possible, and water is a common solvent in many pharmaceutical formulations. There is no reason that the model cannot be applied to totally non-aqueous systems as well. Therefore, it is suggested to be employed in practical applications either for screening experimentally obtained surface tensions for detecting possible outliers or for predicting unmeasured surface tensions of mixed solvents at various temperatures after training using a minimum number of experimental data. The expected mean percentage deviation for the predicted data is $c a$. $8 \%$. The applicability of JAM for correlating surface tension of ternary solvents at various temperatures is also shown and the results revealed its good potential for modeling such data.

Acknowledgment The financial support from Drug Applied Research Center, Tabriz University of Medical Sciences is gratefully acknowledged.

\section{References}

1) Guyton A. C., Hall J. E., "Textbook of Medical Physiology," 9th Ed., Chapters 41-43, W.B. Saunders, Pennsylvania, 1996.

2) Prokop R. M., Chen P., Garg A., Neumann A. W., Col. Sur. B. Biointer., 13, 59-73 (1999).

3) Callaghan C., Barry P., Thorax, 52, 31-44 (1997).
4) Acree W. E., Jr., Thermochim. Acta, 198, 71-79 (1992).

5) Jouyban-Gh. A., Valaee L., Barzegar-Jalali M., Clark B. J., Acree W. E., Jr., Int. J. Pharm., 177, 93-101 (1999).

6) Jouyban-Gh. A., Khaledi M. G., Clark B. J., J. Chromatogr. A, 868 , $277-284(2000)$

7) Jouyban A., Grosse S. C., Chan H. K., Coleman M. W., Clark B. J., J. Chromatogr. A, 994, 191-198 (2003).

8) Jouyban A., Khoubnasabjafari M., Chan H. K., Altria K. D., Clark B. J., Chromatographia, 57, 191-196 (2003).

9) Jouyban A., Chan H. K., Barzegar-Jalali M., Acree W. E., Jr., Int. J. Pharm., 243, 167-172 (2002)

10) Jouyban A., Chan H. K., Clark B. J., Acree W. E., Jr., Int. J. Pharm., 246, 135-142 (2002).

11) Jouyban A., Soltanpour Sh., Chan H. K., Int. J. Pharm., 269, 353360 (2004).

12) Jouyban A., Fathi-Azarbayjani A., Barzegar-Jalali M., Acree W. E., Jr., Pharmazie, (2004) in press.

13) Jouyban-Gh. A., Hanaee J., Int. J. Pharm., 154, 245-247 (1997)

14) Connors K. A., Wright J. L., Anal. Chem., 61, 194-198 (1989).

15) Hoke B. C., Patton E. F., J. Chem. Eng. Data, 37, 331-333 (1992).

16) Lee J.-W., Park S. B., Lee H., J. Chem. Eng. Data, 45, 166-168 (2000).

17) Howard K. S., McAllister R. A., AIChE J., 3, 325-329 (1957).

18) Vazquez G., Alvarez E., Navaza J. M., Rendo R., Romero E., J. Chem. Eng. Data, 42, 57-59 (1997).

19) Alvarez E., Cancela A., Maceiras R., Navaza J. M., Taboas R., J. Chem. Eng. Data, 48, 32-35 (2003).

20) Ramkumar D. H. S., Kudchadker A. P., J. Chem. Eng. Data, 34, 463 465 (1989).

21) Vazquez G., Alvarez E., Rendo R., Romero E., Navaza J. M., J. Chem. Eng. Data, 41, 806-808 (1996).

22) Hovorka F., Schaefer R. A., Dreisbach D., J. Am. Chem. Soc., 58, 2264-2267 (1936)

23) Vazquez G., Alvarez E., Navaza J. M., J. Chem. Eng. Data, 40, 611614 (1995)

24) Weast R. C., "Handbook of Chemistry and Physics," 53rd ed., CRC Press, Ohio, 1972, p. F-29.

25) Alvarez E., Rendo R., Sanjurjo B., Sanchez-Vilas M., Navaza J. M., J. Chem. Eng. Data, 43, 1027-1029 (1998). 